\title{
Household Environmental Tobacco Smoke and Risk of Allergic Sensitization Among Children with Asthma
}

\author{
Căpîlna Brîndușa, Baghiu Maria Despina \\ Pediatric Clinic 1, University of Medicine and Pharmacy, Tîrgu Mureș, Romania
}

\begin{abstract}
Background: Exposure to cigarette smoke is causing health problems, its components are known to possess carcinogenic, mutagenic, cytotoxic or irritant properties. Prevalence of smoking in pregnant women is between $17 \%$ and $35 \%$ worldwide. Passive smoking is identified as a factor with negative impact on health, and children are especially vulnerable. Children raised in families with smokers have a higher incidence of respiratory infections, recurrent wheezing, bronchitis, nocturnal cough and asthma. The aim of this study was determination of sensitization to various allergens in children exposed to cigarette smoke compared with children not exposed to cigarette smoke.

Material and method: One-hundred eighty children treated in Pediatric Clinic 1 of Tîrgu Mureș were included in the study between 20082011. The patients were divided into two groups: 50 children exposed to cigarette smoke and 130 children not exposed. Measurement of lung function was performed in children over 4 years using a spirometer. Serum specific lgE was analyzed to inhalatory and food allergens. Wheezing phenotype was determined in children younger than 4 years and exposure to cigarette smoke was evaluated based on parents' responses to questionnaires.

Results: There was a significantly greater likelihood of developing sensitivity in children exposed to tobacco smoke than in those not exposed to dermatophagoides pteronissimus, dermatophagoides farinae, milk and grass pollen.

Conclusions: During early childhood both pre- and postnatal tobacco smoke exposure has an adjuvant effect on allergic sensitization inhalatory and food allergens.
\end{abstract}

Keywords: smoke exposure, children, allergens

Received: 14 January 2013

\section{Introduction}

Exposure to cigarette smoke is causing health problems worldwide [3]. Tobacco is made from dried leaves of tobacco plants. Tobacco history began 5000 years BC, and is of Native American origin. Christopher Columbus brought tobacco seeds for the first time in Europe (Portugal and Spain) from the West Indies in 1492. Jean Nicot, the French ambassador in Paris planted the seeds in his own garden. In Europe, tobacco was first used for medical purposes (Catherine de Medici was treated for an abdominal pathology). When she received tobacco from Jean Nicot, the French queen named it "nicotiana". Later Linné calls the plant Nicotiana tabacum. Industrial production of cigarettes began in America in the late $19^{\text {th }}$ century. During the two world wars soldiers spread tobacco consumption [4].

Cigarette consumption was originally a male habit. Women have taken the habit during and after World War II, than their children came in close contact with cigarette smoke. Smoking has become a sign of independence for educated and successful women. An other social group that has learned this habit, and where the prevalence of smoking is currently the highest, is the group of poorly educated women [5].

Cigarette smoke is a complex mixture of more than 4800 different components [1]. Many of them are known

Correspondence to: Brîndușa Căpîna

E-mail: brindusarc@yahoo.com to possess carcinogenic, mutagenic, cytotoxic or irritant properties. Constituents of cigarette smoke are polycyclic aromatic hydrocarbons, N-nitrosamines or free radicals, aromatic amines, aldehydes and metals such as nickel, chromium and cadmium [1].

Prevalence of smoking in pregnant women is between $17 \%$ and $35 \%$ worldwide [2]. The prevalence of smoking during pregnancy has declined in recent years, but a significant proportion $(13-16 \%)$ of women continues to smoke during pregnancy in the Nordic countries, and this is influenced by maternal age, ethnicity, educational level and socio-economical factors $[2,6]$.

In Romania, the prevalence of smoking in 1995 was $25 \%$ for women and $43.45 \%$ in general (adults 25-44 years) [7]. Passive smoking is identified as a factor with negative impact on health, and children are especially vulnerable.

\section{Aim of the study}

Determination of sensitization to various allergens in children exposed to cigarette smoke compared with children not exposed to cigarette smoke.

\section{Material and methods}

One-hundred eighty children with asthma treated in Pediatric Clinic 1 in Tîrgu Mureș were included in the study between 2008-2011.

For determination of sensitization to various allergens in children exposed to cigarette smoke compared with chil- 
dren not exposed to cigarette smoke, two groups were created: a group of 50 children exposed to cigarette smoke, and a group of 130 children not exposed to cigarette smoke.

Measurement of lung function was performed in children over 4 years. Peak expiratory flow measurement was performed using a spirometer (Koko Legend Portable Spirometer, Nspire Health, UK). Highest values of forced expiratory volume in 1 second (FEV1) and forced vital capacity (FVC) were extracted and used in the analysis, if the child's effort was maximum, the test curve have passed visual inspection and two higher values were reproducible according to the criteria of the American Thoracic Society and European Respiratory Society (ATS/ERS).

Allergic sensitization was evidenced by measuring serum specific $\operatorname{IgE}$ of patients. Specific IgE antibodies were analyzed to inhaled and food allergens (Synlab Germany) in a combined pediatric panel, comprising respiratory and food allergens. Each allergen sensitization was defined as serum IgE antibody level $\geq 0.35$ kilounits antibody/liter (KUA/L).

Wheezing phenotype was determined in children younger than 4 years using a questionnaire, clinical examination and bronchodilatation test.

\section{Assessment of exposure to cigarette smoke}

The study focused on exploring the effect of cigarette smoke exposure on allergic sensitization in pediatric patients diagnosed with bronchial hyperreactivity. Information related to exposure to cigarette smoke was based on parents' responses to questionnaires.

Postnatal exposure to cigarette smoke was defined as one or more cigarettes smoked per day in the child's habitual space by one of the persons with whom the child lives. These data were collected through a questionnaire, which took into account the activities that avoid direct exposure of children to tobacco smoke: smoking in the yard, on the balcony outside the home, etc.

Statistical analysis of the data was performed using GraphPad Prism. Analysis of the relationship between two variables and intensity of this connection was made with the Pearson correlation coefficient $r$. To see if there is an
Table I. Sensitization to environmental allergens in children exposed to cigarette smoke compared with children not exposed

\begin{tabular}{|c|c|c|c|c|c|c|c|}
\hline \multirow[t]{2}{*}{ Allergen } & \multirow{2}{*}{$\begin{array}{l}\text { Exposed/ } \\
\text { non-exposed } \\
\text { to cigarette } \\
\text { smoke }\end{array}$} & \multicolumn{3}{|c|}{ Allergy } & \multirow[t]{2}{*}{$p$ value } & \multirow[t]{2}{*}{ OR } & \multirow[t]{2}{*}{$95 \% \mathrm{Cl}$} \\
\hline & & Yes & No & Total & & & \\
\hline \multirow[t]{2}{*}{ Derm. Pter. } & Exposed & 27 & 23 & 50 & 0.0007 & 3.3 & $1.67-6.5$ \\
\hline & Non-exposed & 34 & 96 & 130 & & & \\
\hline \multirow[t]{2}{*}{ Derm. Far. } & Exposed & 20 & 30 & 50 & 0.02 & 2.3 & $1.15-4.6$ \\
\hline & Non-exposed & 29 & 101 & 130 & & & \\
\hline \multirow[t]{2}{*}{ Mold } & Exposed & 13 & 37 & 50 & 0.13 & 1.93 & $0.87-4.26$ \\
\hline & Non-exposed & 20 & 110 & 130 & & & \\
\hline \multirow[t]{2}{*}{ Cat } & Exposed & 8 & 42 & 50 & 0.17 & 2.06 & $0.77-5.47$ \\
\hline & Non-exposed & 11 & 119 & 130 & & & \\
\hline \multirow[t]{2}{*}{ Dog } & Exposed & 5 & 45 & 50 & 0.35 & 1.69 & $0.52-5.45$ \\
\hline & Non-exposed & 8 & 122 & 130 & & & \\
\hline
\end{tabular}

association between exposure to tobacco smoke and sensitization to various allergens, a $2 \times 2$ contingency table was used applying the chi square test. Estimated risk, OR (odds ratio) was calculated and values of $p<0.05$ were considered to be statistically significant.

\section{Results}

Sensitization to environmental allergens in those exposed to cigarette smoke compared to those non-exposed was studied for allergens such as Dermatophagoides pteronissimus, Dermatophagoides farinae, mold, dog, cat allergens, grass pollen artemisia pollen, egg, milk and peanuts.

According to our results, there is a 3.3 times greater likelihood of developing Dermatophagoides pteronissimus sensitivity ( $\mathrm{p}=0.0007$ ), and a 2.3 times greater likelihood of developing Dermatophagoides farinae sensitivity in children exposed to tobacco smoke, compared to those not exposed $(\mathrm{p}=0.02)$. No significant association was identified for mold, cat or dog.

\section{Discussions}

When air with cigarette smoke enters in the lungs of children, lung function is impaired by a local inflammatory response, causing epithelial injury, fibrosis and the small airway remodeling [1]. Particular attention is given to in-
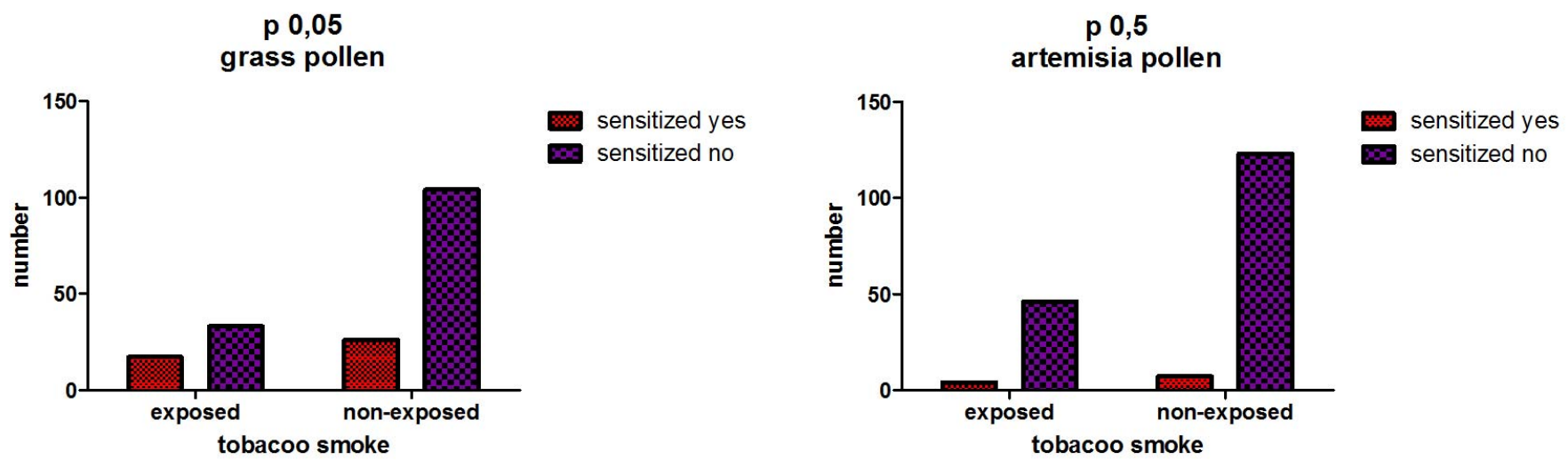

Fig. 1. Sensitization to grass pollen and artemisia pollen in children exposed to cigarette smoke compared with children not exposed 

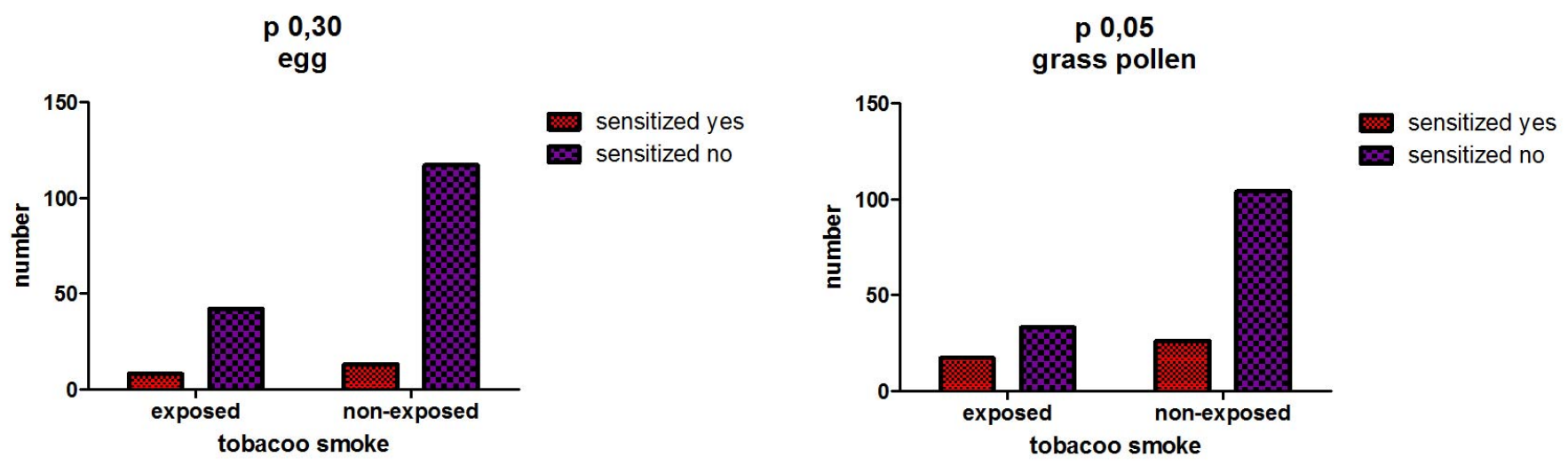

Fig. 2. Sensitization to egg and peanut in children exposed to cigarette smoke compared with children not exposed

trauterine exposure to cigarette smoke, where the harmful effect is mediated by systemic factors directly transmitted from the mother to the fetus [8]. It has been shown that in utero exposure to cigarette smoke leads to oxidative stress, as seen in the blood tests of newborns and infants 3 months of age $[9,10]$.

Children raised in families with smokers have a higher incidence of respiratory infections, recurrent wheezing, bronchitis, nocturnal cough and asthma [1]. Maternal smoking and passive exposure of children to tobacco smoke is also associated with respiratory distress and wheezing $[1,11,12]$. When exposure occurs very early in life, it is almost impossible to separate prenatal exposure and early postnatal exposure [12].

The relationship between parental smoking and asthma was focused on the study of postnatal exposure to maternal or paternal smoking, combined or separately.

Literature data shows that both pre- and postnatal tobacco smoke exposure during early childhood has adjuvant effect on allergic sensitization to food allergens [13]. Our study shows a 2.44 times greater probability of developing sensitization to milk in children exposed to tobacco smoke than those not exposed $(p=0.01)$. No such association for the consumption of eggs or peanuts $(\mathrm{p}=0.30$, and $\mathrm{p}=$ 0.15 , respectively) was found.

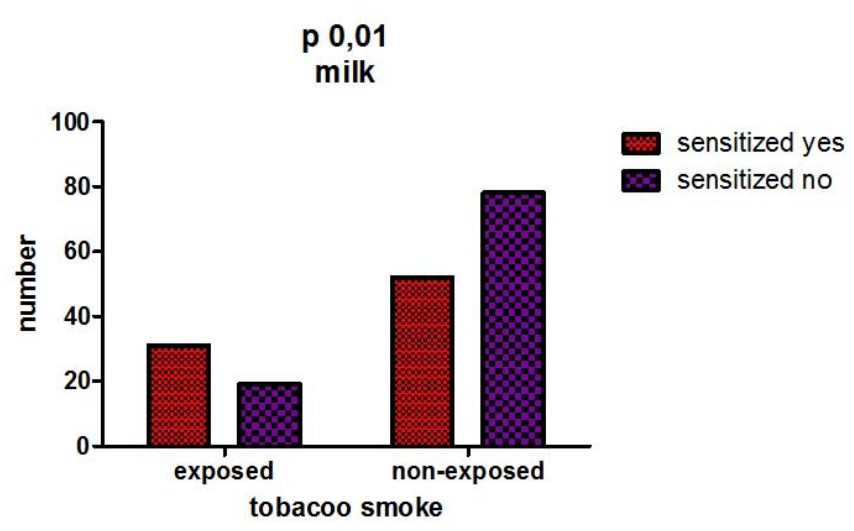

Fig. 3. Sensitization to milk in children exposed to cigarette smoke compared with children not exposed
In a study carried out by Lannerö $\mathrm{E}$ et al. it is described that a dose-response effect exists for exposure to environmental tobacco smoke (ETS) from parental smoking during the first months of life and IgE sensitisation. There was an increased risk of sensitisation to inhalation and/or food allergens [OR (adj) 1.28 (95\%CI 1.01-1.62)] among children exposed to ETS at 2 months of age. The IgE sensitising effect of ETS seemed to be confined to infants of parents without allergic diseases and to ETS exposure during early infancy [14].

In our study we found a 3.3 times greater likelihood of developing sensitization to Dermatophagoides pteronissimus and a 2.3 times greater likelihood of developing sensitization to Dermatophagoides farinae in children exposed to smoke than those not exposed. No significant associations were identified for mold, cat, dog, egg or peanuts allergens. The 2.06 times higher probability to develop sensitization to grass pollen in children exposed to tobacco smoke than those not exposed $(\mathrm{p}=0.05)$ is comparable with data found in the literature. In contrast, no significant association was identified for artemisia pollen $(p=0.50)$.

\section{Conclusions}

Our results indicate that exposure of children to specific air pollution, namely cigarette smoke, increases the risk of IgE sensitization to environmental allergens and food allergens.

The best way to protect children from exposure to tobacco smoke, except smoking ban, is smoking outside of the home, behind closed doors. Society, including the authorities, must make further efforts to train and support smoker parents who smoke in their homes.

\section{References}

1. Lee JP, Battle RS, Lipton R, Soller B. Smoking: use of cigarettes, cigars and blunts among Southeast Asian American youth and young adults. Health Educ Res. 2010;25(1):83-96.

2. Goodman J. Tobacco in History: The Cultures of Dependence. Routledge, London and New York, 1995, 80-81.

3. Graves B. Tabacco use. Capstone Press, Minnesota, 2000, 14-19.

4. Bhalla DK, Hirata F, Rishi AK et al. Cigarette smoke, inflamation, and long injury: a mechanistic perspective. J Toxicol Environ Health B Crit Rev. 2009;12(1):45-64. 
5. Prescott SL. Effects of early cigarette smoke exposure on early immune development and respiratory disease. Paediatric Breathe Rev. 2008;9(1): 3-9.

6. Kvalvik LG, Skjaerven R, Haug K, Smoking during pregnancy from 1999 to 2004: a study from the Medical Birth Registry of Norway. Acta Obstet Gynecol Scand. 2008;87(3):280-5.

7. WHO Tobacco Control Country Profiles, Second Edition, 2003. Available online at http://www.who.int/tobacco/global_data/country_profiles/ Introduction.pdf

8. Pietinalho A, Pelkonen A, Rytila P. Linkage between smoking and asthma. Allergy 2009;64(12):1722-7.

9. Aycicek A, Ipek A. Maternal active or passive smoking oxidative causes stress in cord blood. Eur J Pediatr. 2008;167(1):81-5.

10. Noakes PS, Thomas R, Lane $\mathrm{C}$ et al. Association of maternal smoking with infant increased oxidative stress at 3 month of age. Thorax. 2007; $62(8): 714-7$.

11. Carlsen $\mathrm{KH}$, Carlsen $\mathrm{KC}$. Respiratory effects of tobacco smoking on Infants and young children. Paediatric Breathe Rev. 2008;9(1):11-9; quiz 19-20.

12. Stocks J, Dezateux C. The effect of smoking on long term parenteral function and development during infancy. Respirology. 2003;8(3):266-85.

13. Kulig M, Luck W, Wahn U. The association between pre- and postnatal tobacco smoke exposure and allergic sensitization during early childhood. Multicenter Allergy Study Group, Germany. Hum Exp Toxicol. 1999;18(4):241-4.

14. Lannerö E, Wickman M, van Hage $M$ et al. Exposure to Environmental Tobacco Smoke and sensitisation in children. Thorax. 2008;63(2):172-6. 